Chapter II

\author{
LOCAL DYNAMO: UBIQUITOUS \\ SMALL-SCALE MAGNETIC \\ FIELDS AND "HIDDEN MAGNETISM"
}




\title{
Solar magneto-convection
}

\author{
Manfred Schüssler \\ Max-Planck-Institut für Sonnensystemforschung \\ 37191 Katlenburg-Lindau, Germany
}

\begin{abstract}
An overview is given about recent developments and results of comprehensive simulations of magneto-convective processes in the near-surface layers and photosphere of the Sun. Simulations now cover a wide range of phenomena, from whole active regions, over individual sunspots and pores, magnetic flux concentrations and vortices in intergranular lanes, down to the intricate mixed-polarity structure of the magnetic field generated by small-scale dynamo action. The simulations in concert with high-resolution observations have provided breakthroughs in our understanding of the structure and dynamics of the magnetic fields in the solar photosphere.
\end{abstract}

Keywords. Sun: magnetic fields, Sun: photosphere, convection, (magnetohydrodynamics:) MHD

\section{Introduction}

Solar magneto-convection is a rather extended area of research. It covers topics ranging from the generation of the large-scale magnetic field by the solar dynamo in the deep convection zone to the excitation, propagation, and dissipation of currents and MHD waves in the chromosphere and beyond. Obviously, this wide field cannot be reasonably covered in a single review. Recent reviews of various aspects of solar magneto-convection can be found in Fan (2009), Charbonneau (2010), Stein (2012), and Weiss (2012).

Here I shall restrict the discussion to the solar photosphere, the region where the best understanding of the magneto-convective processes has been achieved so far. The significant progress was made possible through the interplay between high-resolution observations, idealized theoretical models, and comprehensive numerical simulations. The latter provide a $3 \mathrm{D}$ view of the processes underlying the observations. Simulations can be validated (albeit not in a strict sense) by detailed comparison of synthetic observational quantities based on simulation results with real observations. At the same time, this comparison provides a firm basis for the interpretation of the observations in terms physical quantities and processes.

I prefer to qualify these simulations as 'comprehensive' in favor of the often-used term 'realistic', the reason being that the values of important non-dimensional numbers (such as the kinetic and magnetic Reynolds numbers, or the magnetic Prandtl number) that can be reached in the simulations differ from the realistic values by (many) orders of magnitude. Typically, some kind of hyperdiffusion or subgrid modelling is used to account for the unresolved spatial scales, but in the absence of a theory of turbulence the validity of such quasi-heuristic approaches cannot be strictly proven. On the other hand, the term 'comprehensive' expresses the fact that these simulations aim at representing all relevant physical processes and conditions in the photosphere, i.e., compressible MHD in 3D domain, partial ionization and molecule formation, as well as non-grey and nonlocal radiative transfer. A number of numerical codes are used to obtain comprehensive simulations, among them the ANTARES code (Muthsam et al. 2010), the CO5BOLD code (Freytag et al. 2012), the MURaM code (Vögler 2003; Vögler et al. 2005), the PENCIL code (Brandenburg \& Dobler 2002), the STAGGER/BIFROST code (Gudiksen et al. 2011), as well as the codes of Robinson et al. (2003), and Jacoutot et al. (2008). 
There are also codes with rather coarse treatment of radiation, such as the RADMHD code (Abbett 2007; Abbett \& Fisher 2012) with a local radiative cooling term or the code of Ustyugov (2010), which uses the diffusion approximation. Unfortunately, not all groups have given detailed descriptions of the numerical methods and approximations used in their codes and shown test results in the literature, so that it is difficult to judge and trust the validity of their simulation results. On the other hand, the STAGGER, CO5BOLD and MURaM groups groups have embarked on a cross-validation of their codes by a detailed comparison of numerical results (Beeck et al. 2012).

Even when restricting oneself to the photosphere, a complete review of observational, theoretical, and simulation results is far beyond the scope of this contribution. Limitations of space (and human time) force me to restrict myself mainly to the discussion of recent simulation results, with very limited references to observation and theoretical concepts. Furthermore, I am afraid that this text is somewhat biased towards results obtained with the MURaM code - not because this code would be better than others, but just because I am most familiar with its results. What follows is organized according to the spatial scale of the phenomenon: from whole active regions down to the fine threads of the magnetic field generated by small-scale dynamo action.

\section{Active regions}

In the past decade, the computational resources available for comprehensive simulations have increased dramatically. It has become possible to simulate the emergence and development of whole active regions with sunspots, pores, and plage areas. This line of research was started by Cheung et al. (2007), who considered the emergence of a twisted flux tube carrying a longitudinal magnetic flux of $10^{19} \mathrm{Mx}$. The initially coherent flux tube becomes undulated and fragmented by the vigorous convective flows, so that the flux emergence takes place in the form of numerous patches of horizontal field connecting small bipoles in a 'salt-and-pepper' pattern. The disturbed granulation at the emergence site shows elongated granules threaded by dark lanes. Many of these features are also found in observations (e.g., Cheung et al. 2008; Yelles Chaouche et al. 2009). Similar results were found by Stein et al. (2011), Fang et al. (2010, 2012), and by Martínez-Sykora et al. $(2008,2009)$. The latter authors also considered the evolution of the coronal field during flux emergence and the development of spicule-like structures.

Cheung et al. (2010) simulated the emergence of a flux tube with $7.6 \times 10^{21} \mathrm{Mx}$. As the emergence proceeds, the pattern of small bipoles at the visible solar surface increasingly reorganizes until the magnetic flux distribution reflects the large-scale bipolar structure of the underlying Omega-shaped flux tube. At the same time, a pair of sunspots forms through the coalescence of pores and smaller flux fragments, so that the appearance of a full-fledged active region develops. These simulations also solved the long-standing problem of how the enormous amount of mass contained in a deep-lying flux tube is removed as it reaches the low-density regions near the surface. It turns out that much of this mass is accumulated in sinking U-shaped loops that reconnect near the surface and thus release the mass from the field lines of the surface field. Observational evidence for this process has recently been reported (Centeno 2012). Simulations by M. Cheung and M. Rempel (not yet published) show that an initial twist is not essential for the formation of coherent sunspots from a flux tube inserted at a depth of about $16 \mathrm{Mm}$.

While the simulations discussed so far assumed a large-scale flux tube to coherently enter the simulation domain from below, Stein \& Nordlund (2012) assumed horizontal magnetic field to be advected by upflows at the bottom boundary of the computational domain. They found the development of a bipolar structure at the surface that resembled 
a small active region with a few pores. Although this simulation shows that a bipolar region can form without inserting a coherent flux tube beforehand, some caution is indicated when interpreting this result: the horizontal field advected into the computational box has a fixed direction and strength, so that the simulation implicitely assumes the existence of an infinite reservoir of strictly organized and coherent horizontal magnetic flux below the computational domain, in fact something like a huge homogeneous flux sheet. Consequently, the orientation of the developing bipolar region is along the direction of the assumed horizontal field below the simulation box.

\section{Sunspots and pores}

Comprehensive simulations of sunspots have seen tremendous progress in the last few years. Already studies of 'sunspot slabs' at rather coarse resolution and limited box size (Heinemann et al. 2007; Rempel et al. 2009b) provided crucial insights in the processes responsible for the penumbral filamentation and the Evershed effect. The first simulations of full sunspots by Rempel et al. (2009a) and their further development and detailed analysis by Rempel (2011a,b, 2012) revealed the nature of the magneto-convective processes leading to the characteristic structure of sunspots. While the bright umbral dots represent hot upflow plumes, whose lateral expansion is strongly constricted by the vertical umbral field (Schüssler \& Vögler 2006; Bharti et al. 2010), the inclined field in the penumbra provides a path for the unimpeded expansion of hot upflowing material: the direction along the field. As a consequence, sheet-like convective upflows in the penumbra appear as bright, elongated filaments with strong outflows (representing the Evershed effect), which feed downflow patches in the outer part of the penumbra. Part of the rising plasma turns over laterally (perpendicular to the filament axis) and descends in downflow lanes next to the bright filaments. The expanding outflow stretches and expels the magnetic field, so that the outflow channels exhibit relatively weak (albeit non-vanishing) and almost horizontal magnetic field. All these properties are consistent with observational results (e.g., Borrero \& Ichimoto 2011; Rempel \& Schlichenmaier 2011; Joshi et al. 2011; Scharmer et al. 2011).

It is observationally well established that the formation of big sunspots invariably is connected to major events of magnetic flux emergence. This is also reflected in simulations of sunspot formation and structure, which always require either the advection of well-organized magnetic flux into the computational domain (as in the simulations of active-region formation discussed in Sec. 2) or the assumption of a sufficiently big concentration of vertical magnetic flux as initial condition for the simulation. The situation is different with pores, small dark flux concentrations without penumbrae and mostly not exceeding the area of a few granules. Such structures are observed to form and decay quasi-spontaneously in mature plage areas, without any associated emergence of new magnetic flux. Magneto-convection simulations in sufficiently deep boxes also show the formation of pore-like dark structures if enough magnetic flux is available, either in the form of a preset vertical flux through the computational box (Kitiashvili et al. 2010; Ustyugov 2010) or by the perpetual advection of horizontal magnetic flux through the bottom boundary (Stein et al. 2011). Previous simulations in shallow boxes extending to a few Mm depth below the visible solar surface did not show the formation of dark structures, apart from occasional small 'micropores' (e.g., Bercik et al. 2003; Vögler et al. 2005; Stein \& Nordlund 2006). The reason why shallow simulations miss the formation of proper pores is that it requires horizontal flows on a sufficiently big spatial scale in order to collect the necessary sufficient amount of magnetic flux. Since the horizontal scale of 

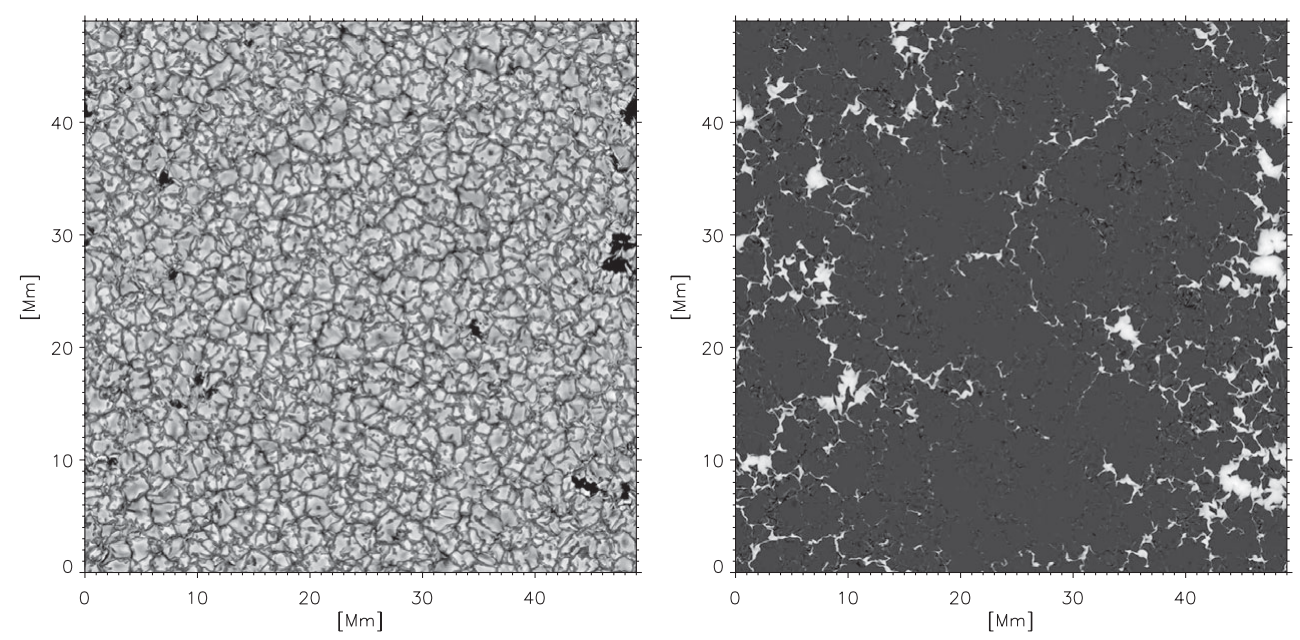

Figure 1. Maps of brightness (bolometric intensity, left panel) and vertical magnetic field at the optical solar surface (right panel) from a snapshot of a magneto-convection simulation in a $49 \times 49 \mathrm{Mm}^{2}$ wide and $15 \mathrm{Mm}$ deep box permeated by a horizontally averaged vertical field of $100 \mathrm{G}$.

the convective flows increases with depth (see, e.g., Fig. 6 in Nordlund et al. 2009), only sufficently deep boxes (at least $6 \mathrm{Mm}$ ) provide the conditions for pore formation.

A word of caution is in order here. The maximum horizontal scale of the convective motions seen in simulations is roughly equal to the depth. This means that the horizontal extension of the computational box should be at least 3-4 times its depth in order to provide enough volume for a development of a large-scale flow pattern that is not restrained by the periodic bounday conditions at the side walls of the box. Simulation in boxes with smaller aspect ratios most probably provide unrealistic results.

What determines the size (or amount of flux) of pores and why do big sunspots not form in this way? This depends basically on the amount of magnetic flux available (mean flux density) and on the relation of the advection time of the horizontal flow (horizontal scale divided by flow speed) and the lifetime of the flux concentration: the advected flux per unit time has to be at least equal to the flux lost by 'turbulent erosion' of the flux concentration (Cameron et al. 2007). As a result, for the mean vertical flux densities found in plages (a few hundred $\mathrm{G}$ ), the biggest flux concentrations formed by flux advection do not exceed the size of a few granules. As an example, Fig. 1 shows maps of the emergent intensity (left panel) and of the surface distribution of vertical magnetic field (right panel) from a simulation (run by M.C.M. Cheung) in a box of $49 \times 49 \mathrm{Mm}^{2}$ horizontal extension reaching down to $15 \mathrm{Mm}$ depth, so that nearly supergranular-size flows can develop. The average vertical field strength is $100 \mathrm{G}$, thus representing a typical plage region. The simulation formed a number of pores, which qualitatively resemble those occuring in plage areas. Furthermore, the distribution of the magnetic field shows a multi-cellular pattern, reflecting the various horizontal scales of the convective flow. The biggest cells have a scale of about $15 \mathrm{Mm}$, corresponding to the depth of the computational box. This pattern is remarkably similar to those seen in plage observations. This result opens the possibility to obtain information about the flows in the upper part of the convection zone by surface observations and comparison with simulations, providing a supplement to helioseismic inversions.

Fig. 2 shows the distribution of the vertical magnetic field component on a vertical section of the simulation box. The section cuts through surface flux concentrations of 


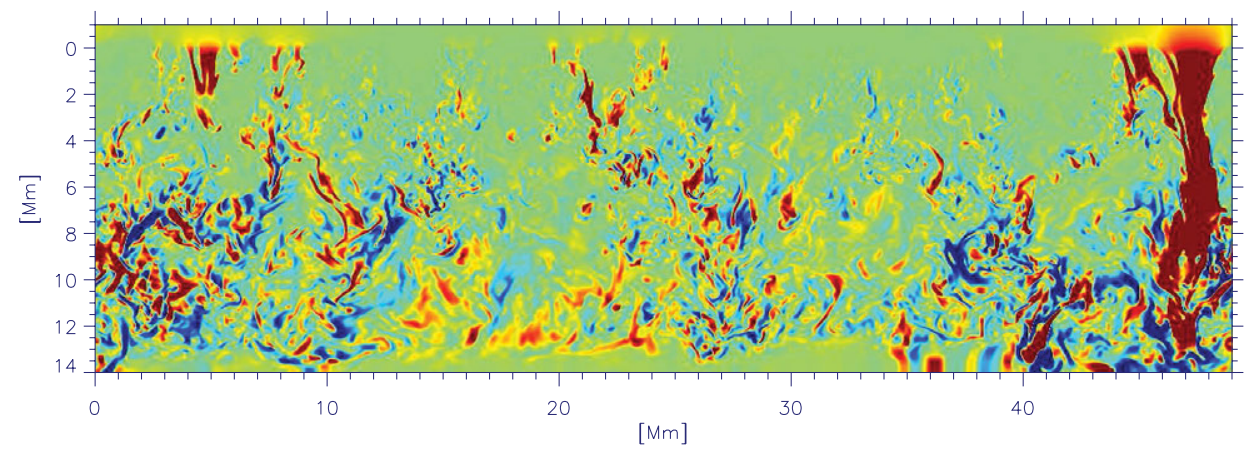

Figure 2. Map of the vertical magnetic field component, $B_{z}$, in a vertical cut along a horizontal line at $x=27.5 \mathrm{Mm}$ in Fig. 1 (right panel). Reddish colors indicate positive values of $B_{z}$, bluish colors negative values. The zero level of the depth scale corresponds to the optical surface (mean level of optical depth unity).

various sizes. While the small-scale flux concentrations in intergranular lanes are restricted to the near-surface layers, bigger flux concentrations extend deeper, reflecting the depth of the flow pattern that accumulated them in the first place. The big magnetic flux concentration corresponding to the pore near the right-hand edge of the plot stretches down almost to the bottom of the computational domain. While the magnetic field is rather organized in the surface layers, the deeper parts of the box show patches with a chaotic pattern of small-scale field structures, resulting from the turbulent nature of the convective downflow regions. These are the sites of small-scale dynamo action (see Sec. 6).

\section{Small-scale flux concentrations}

Starting with the pioneering work of Nordlund (1983, 1985b), comprehensive 3D simulations have revealed the processes responsible for the concentration of most of the 'non-turbulent' part of the magnetic flux at the solar surface into small-scale structures with kG field strength located in intergranular downflow lanes (e.g., Steiner et al. 1996, 1998; Bercik et al. 1998; Stein et al. 2002; Vögler \& Schüssler 2003; Vögler et al. 2005; Schaffenberger et al. 2006; Stein \& Nordlund 2006). The simulations confirmed the validity of the basic theoretical explanations for the intermittent structure of the solar surface field, which were developed already in the 60 s and 70 s of the last century. The first concept is flux expulsion by convective flows in an electrically conducting fluid, pioneered by Weiss $(1964,1966)$ and Parker $(1963)$. The advection of magnetic flux by horizontal flows and its concentration in convective downflow areas concentrates the field roughly up to equipartition field strength, for which the magnetic energy density equals the kinetic energy density of the flow. The concept of convective collapse is based in the strongly superadiabatic startification of the subsurface layers, which renders equipartition flux concentrations unstable with respect to downflow along the field lines (Webb \& Roberts 1978; Spruit \& Zweibel 1979). This evacuates the upper part of the flux concentrations, so that the gas pressure of the surrounding plasma compresses the flux concentration until kG field strength is reached (Parker 1978; Spruit 1979). The depression of the surface of optical depth unity in the evacuated flux concentration leads to lateral heating of its interior by the hot walls and its appearance as a bright structure (Spruit 1976; Deinzer et al. 1984). Quantitative comparison of observations and comprehensive numerical simulations confirmed the relation between $\mathrm{kG}$ magnetic flux concentrations and 
bright points (Schüssler et al. 2003; Shelyag et al. 2004; Lagg et al. 2010; Röhrbein et al. 2011; Danilovic et al. 2012), faculae (Keller et al. 2004; Carlsson et al. 2004), and the spectro-polarimetric signatures of these structures (Shelyag et al. 2007).

\section{Vortices}
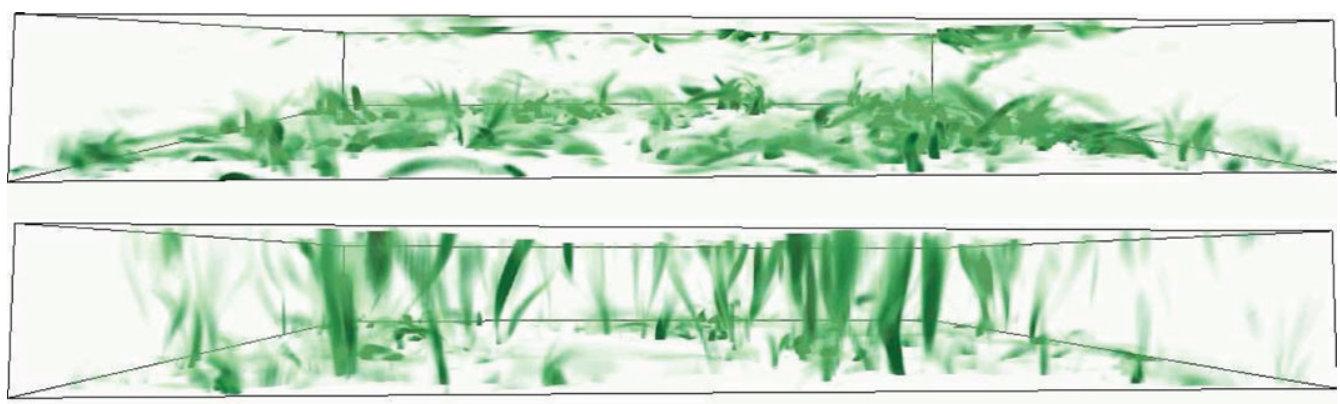

Figure 3. Volume rendering of the swirling strength (for a definition, see Moll et al. 2011a) for a non-magnetic simulation (upper panel) and for a simulation with an average vertical magnetic field of $200 \mathrm{G}$ (lower panel), viewed from one side of the computational box. Shown is the upper half $\left(6 \times 6 \times 0.8 \mathrm{Mm}^{3}\right)$ of the box, the bottom plane corresponding roughly to the optical surface. While the vertical vortex tubes turn over near the surface in the non-magnetic case, they align with the vertical flux concentration and protrude high into the atmosphere when a background magnetic field is present.

The formal equivalence of the vorticity equation and the induction equation suggests that vertical vorticity should be expelled and concentrated in intergranular downflows in similar ways as magnetic flux. It is no surprise, therefore, that vortical features and whirl flows are regularly found in comprehensive convection simulations (Nordlund 1985a; Stein \& Nordlund 1998; Moll et al. 2011a; Kitiashvili et al. 2012a). While most of the strong vertical vortices have diameters below $100 \mathrm{~km}$, probably too small to be detected with existing telecopes, larger-scale vortex flows have been reported by observers (e.g., Brandt et al. 1988; Bonet et al. 2008, 2010; Attie et al. 2009; Vargas Domínguez et al. 2011). In addition, Steiner et al. (2010) detected horizontal vortices in granules, both in simulations and in observations with the Sunrise balloon telescope.

If a sufficient amount of vertical background flux is present, the vortices are closely associated with magnetic flux concentrations. Vögler (2004) first found 'magnetic vortices' in magneto-convection simulations. Recently, this topic found considerable interest, also in connection with chromospheric and coronal heating processes (e.g., Shelyag et al. 2011; Kitiashvili et al. 2012b; Steiner \& Rezaei 2012; Wedemeyer-Böhm et al. 2012). The simulations of Moll et al. (2012) indicate that the dynamical structure of the upper photosphere and chromosphere of 'quiet' regions (i.e., areas with a mean signed field below about $20 \mathrm{G}$ ) is significantly different from that of more strongly magnetized regions: while the former are dominated by patterns of moving shock fronts (as noted previously by Wedemeyer et al. 2004; Wedemeyer-Böhm 2010), the more magnetized areas show numerous vertical vortices, which are strongly associated with the small-scale magnetic flux concentrations in intergranular lanes. As illustrated in Fig. 3, vertically orientated vortices in the surface layers of weakly magnetized regions do not extend high into the atmosphere, but turn over near the optical surface and form low-lying vortex loops (Moll et al. 2011a). In more strongly magnetized regions (network, plage), the vertical vortices align with the magnetic flux concentrations and reach high into the atmosphere, where 
the dissipation of kinetic energy leads to intense local heating. Fig. 4 illustrates the different dynamical character of the upper photosphere/lower chromosphere in weakly and strongly magnetized regions: while shocks dominate the former, hot vortices provide the governing structure in the latter case.
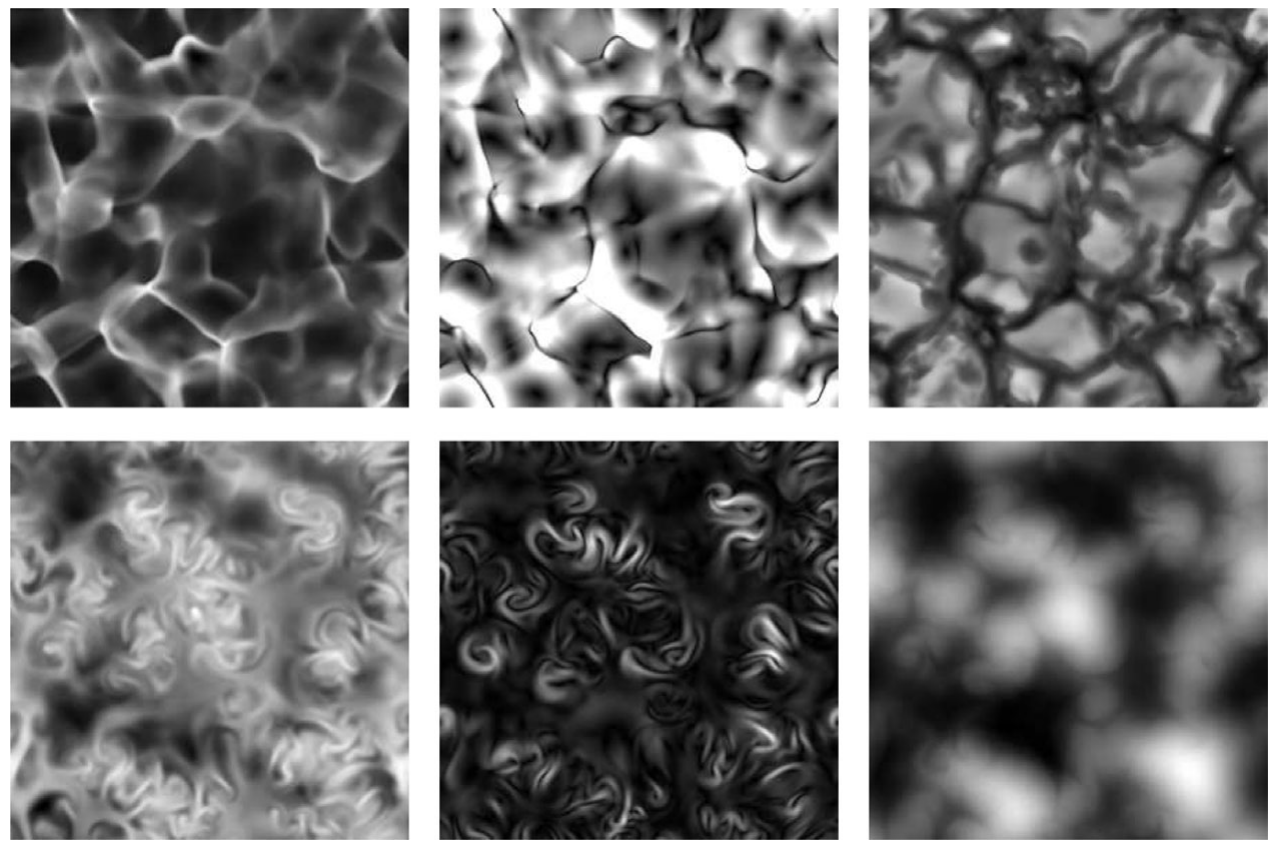

Figure 4. Horizontal cuts (size $6 \mathrm{Mm} \times 6 \mathrm{Mm}$ ) through the upper photospheric layers of a nonmagnetic simulation (upper row) and a simulation with an average vertical magnetic field of $200 \mathrm{G}$ (lower row). Shown are maps of temperature (left panels), ranging from about $3000 \mathrm{~K}$ to over $7000 \mathrm{~K}$, and of horizontal flow speed (middle panels), which reaches values up to $15 \mathrm{~km} \cdot \mathrm{s}^{-1}$ ). The upper right panel shows a (bolometric) brightness map for the hydrodynamic case and the lower right panel gives a map of the vertical magnetic field in the upper photosphere of the magnetic run. The hydrodynamic case is dominated by shock fronts while vortices prevail in the magnetic simulation. Both kinds of structures show strong local heating of the plasma.

\section{Small-scale dynamo}

There are a number of observational indications for the existence of a considerable amount of magnetic flux in the form of a small-scale turbulent field in the solar nearsurface layers (Sánchez Almeida \& Martínez González 2011, see also the contribution of S. Tsuneta in this volume). This component of the solar surface flux seesm to be independent of the 11-year solar cycle (e.g., Trujillo Bueno et al. 2004), indicating a different source mechanism. Recently, D. Bühler (in preparation) systematically analyzed Hinode/SP data for the period between 2007 and 2012, which covers the extended solar minimum and the rise of the current cycle. He found that the area fraction of weak, but significant, linear and circular polarization signals in quiet internetwork regions did not change during this time. This suggests that the turbulent magnetic field is not connected to the solar cycle, but possibly generated by small-scale dynamo (SSD) action.

Characteristic for a SSD driven by a turbulent flow in an electrically conducting fluid is that the magnetic field is generated at spatial scales that are much smaller than that of the energy-carrying eddies of the flow (i.e., the integral scale of the turbulence). Although 
there is some dependence on the value of the magnetic Prandtl number (ratio of kinematic viscosity to magnetic diffusivity), SSD action probably occurs in all turbulent flows of sufficiently high magnetic Reynolds number (see Brandenburg et al. 2012, and references therein). While direct numerical simulations demonstrated SSD action in various settings since the 1980s (e.g., Meneguzzi et al. 1981; Cattaneo 1999; Bushby et al. 2012), the effect was also found in large-eddy simulations (Boussinesq or anelastic) in spherical shells carried out to model the solar convection zone (Gilman \& Miller 1981; Glatzmaier 1985; Brun et al. 2004).

Comprehensive simulations of solar near-surface convection indicate that the observed turbulent field could indeed be a product of a SSD action driven by the turbulent intergranular downflows (Vögler \& Schüssler 2007; Pietarila Graham et al. 2010; Moll et al. 2011b). The characteristic properties of a magnetic field generated by a SSD can explain the observed strong horizontal fields observed in the middle photosphere (Schüssler \& Vögler 2008) and the weak signals detected with sensitive polarimeters (Pietarila Graham et al. 2009; Danilovic et al. 2010a,b). While most of the field due to the SSD is of mixed polarity on small scales and has a strength of some tens to a few hundred Gauss, occasionally enough unipolar flux is being assembled by the granular flows to form a $\mathrm{kG}$ flux concentration appearing as a bright point in the visible light. Therefore, observations of such features in quiet internetwork areas on the Sun are consistent with SSD action.
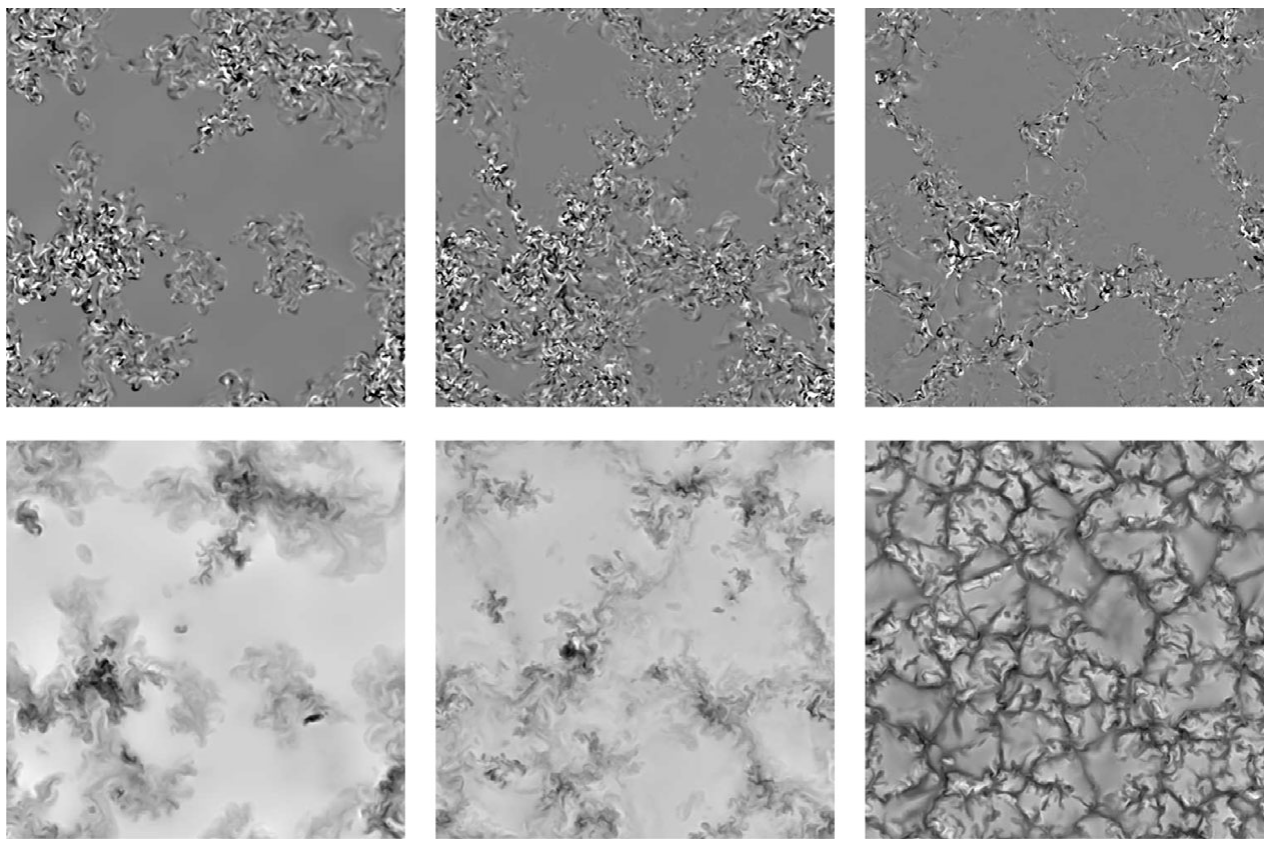

Figure 5. Snapshot from a simulation of small-scale dynamo action in the solar near-surface layers. The computational box is $12 \times 12 \mathrm{Mm}^{2}$ wide and $6.1 \mathrm{Mm}$ deep. The panels show horizontal cuts of the vertical field component (upper panels; black and white indicates negative and positive polarity, respectively) and of the vertical flow velocity (lower panels; light shades indicates upflows, dark shades downflows). The cuts were taken at a depth of $\sim 5 \mathrm{Mm}$ (left panels) and $2.5 \mathrm{Mm}$ (middle panels) below the average height of the optical surface, as well as at the optical surface (right panels). The dynamo-generated field is associated with the downflows in the deeper parts of the domain and thus exhibits a 'mesogranular' pattern at the surface.

Fig. 5 shows a snapshot from a dynamo simulation carried out in a deeper and wider computational box than previous simulations. The maps show a close association between 
the dynamo-generated field, which exhibits the characteristic mixed-polarity pattern of small-scale dynamo action, and downflow areas. This is due to the fact that the small-scale dynamo mainly works in the turbulent downflows. The long-lived, large-scale horizontal convective flow pattern in the deep layers is reflected in the 'mesogranular' distribution of the magnetic field in the surface layers (cf. Yelles Chaouche et al. 2011). The scale of this pattern is determined by the relation between the horiontal advection time and the timescale of flux generation (growth rate of the SSD) in the intergranular downflows.

\section{Concluding remarks and outlook}

The combined efforts of high-resolution observations and comprehensive numerical simulations have tremendously improved our understanding of the magneto-convective processes in the near-surface layers and the photosphere of the Sun. The identification of key physical processes through the simulations and the detailed quantitative comparison between observational data and numerical results was the key factor for this progress. It should be noted that such a comparison needs to be done in a proper way, which means that observational data (e.g., intensity images or Stokes profiles) should be compared with the corresponding quantities derived from the simulation results (i.e., synthetic Stokes profiles) after taking into account instrumental effects (e.g., convolving with point spread functions, adding straylight and noise, rebinning to detector pixel size). Comparing secondary observational products such as inversion results directly with physical quantities from a simulation can lead to misleading conclusions.

Where do we go from here, which are the frontiers of the field? In my opinion, there are three lines of research that will dominate the field in the coming years.

(a) The local comprehensive simulations will make contact to the global simulations of the deep convection zone carried out with anelastic codes. This will require wider and deeper boxes and the inclusion of the effects of sphericity and rotation. By combining the results (or even the codes), a comprehensive understanding of solar convection and its surface manifestations will hopefully be achieved. This is the more important considering recent helioseismic results that suggest an inconsistency with global simulations (Hanasoge et al. 2010, 2012; Gizon \& Birch 2012).

(b) An second important goal is to extend the simulation domain into the chromosphere (and corona) with a proper treatment of the radiation processes. In the chromosphere, the energy balance is dominated by radiative transfer in a few strong spectral lines, all of which are formed in NLTE. In addition, ionization and recombination can no longer be treated as local equilibrium processes. A consistent treatment of these effects within the framework of a 3D MHD simulation provides a considerable challenge, which requires a significant amount of development work (e.g., Gudiksen et al. 2011; Carlsson \& Leenaarts 2012). This also involves the treatment of the Hall effect and ambipolar diffusion in non-ideal MHD (e.g., Cheung \& Cameron 2012; Martínez-Sykora et al. 2012; Khomenko \& Collados 2012).

(c) Finally, the comprehensive simulations have reached a degree of maturity and validation by solar observations that affords their application to other cool stars. 3D hydrodynamic simulations of this kind have been carried out already for some time (e.g., Nordlund \& Dravins 1990; Collet et al. 2007; Ludwig et al. 2009; Freytag et al. 2012). Recently, the first magneto-convection simulations in the surface layers of other cool stars than the Sun have become available (Beeck et al. 2011; Wedemeyer et al. 2012). Such simulations will provide a crucial tool for testing and calibrating methods to infer stellar magnetic fields from observational spectral and spectro-polarimetric data. 


\section{References}

Abbett, W. P. 2007, ApJ, 665, 1469

Abbett, W. P. \& Fisher, G. H. 2012, Sol. Phys., 277, 3

Attie, R., Innes, D. E., \& Potts, H. E. 2009, A\& A, 493, L13

Beeck, B., Collet, R., Steffen, M., Asplund, M., Cameron, R. H., Freytag, B., Hayek, W., Ludwig, H.-G., \& Schüssler, M. 2012, A\& A, 539, A121

Beeck, B., Schüssler, M., \& Reiners, A. 2011, in ASP Conf. Ser. 448, 16th Cambridge Workshop on Cool Stars, Stellar Systems, and the Sun, ed. C. Johns-Krull, M. K. Browning, \& A. A. West, 1071

Bercik, D. J., Basu, S., Georgobiani, D., Nordlund, A., \& Stein, R. F. 1998, in ASP Conf. Ser. 154: Cool Stars, Stellar Systems, and the Sun 10, ed. R. A. Donahue \& J. A. Bookbinder (San Francisco: Astronomical Society of the Pacific), 568

Bercik, D. J., Nordlund, A., \& Stein, R. F. 2003, in ESA Special Publication, Vol. 517, GONG+ 2002. Local and Global Helioseismology: the Present and Future, ed. H. Sawaya-Lacoste, 201-206

Bharti, L., Beeck, B., \& Schüssler, M. 2010, A\& A, 510, A12

Bonet, J. A., Márquez, I., Sánchez Almeida, J., Cabello, I., \& Domingo, V. 2008, ApJ (Letters), 687, L131

Bonet, J. A., Márquez, I., Sánchez Almeida, J., Palacios, J., Martínez Pillet, V., Solanki, S. K., del Toro Iniesta, J. C., Domingo, V., Berkefeld, T., Schmidt, W., Gandorfer, A., Barthol, P., \& Knölker, M. 2010, ApJ (Letters), 723, L139

Borrero, J. M. \& Ichimoto, K. 2011, Living Rev. Solar Phys., 8, 4 http://www.livingreviews.org/lrsp-2011-4

Brandenburg, A. \& Dobler, W. 2002, Comp. Phys. Comm., 147, 471

Brandenburg, A., Sokoloff, D., \& Subramanian, K. 2012, Space Sci. Rev., 169, 123

Brandt, P. N., Scharmer, G. B., Ferguson, S., Shine, R. A., \& Tarbell, T. D. 1988, Nature, 335, 238

Brun, A. S., Miesch, M. S., \& Toomre, J. 2004, ApJ, 614, 1073

Bushby, P. J., Favier, B., Proctor, M. R. E., \& Weiss, N. O. 2012, Geoph. Astrophys. Fluid Dyn., 106,508

Cameron, R., Schüssler, M., Vögler, A., \& Zakharov, V. 2007, A\&AA, 474, 261

Carlsson, M. \& Leenaarts, J. 2012, A\&A, 539, A39

Carlsson, M., Stein, R. F., Nordlund, Å., \& Scharmer, G. B. 2004, ApJ (Letters), 610, L137

Cattaneo, F. 1999, ApJ, 515, L39

Centeno, R. 2012, ApJ, 759, 72

Charbonneau, P. 2010, Living Rev. Solar Phys., 7, 3, http://www.livingreviews.org/lrsp-2010-3

Cheung, M. C. M. \& Cameron, R. H. 2012, ApJ, 750, 6

Cheung, M. C. M., Rempel, M., Title, A. M., \& Schüssler, M. 2010, ApJ, 720, 233

Cheung, M. C. M., Schüssler, M., \& Moreno-Insertis, F. 2007, A\&A, in press

Cheung, M. C. M., Schüssler, M., Tarbell, T. D., \& Title, A. M. 2008, ApJ, 687, 1373

Collet, R., Asplund, M., \& Trampedach, R. 2007, A\&\&A, 469, 687

Danilovic, S., Beeck, B., Pietarila, A., Schüssler, M., Solanki, S. K., Martínez Pillet, V., Bonet, J. A., del Toro Iniesta, J. C., Domingo, V., Barthol, P., Berkefeld, T., Gandorfer, A., Knölker, M., Schmidt, W., \& Title, A. M. 2010a, ApJ (Letters), 723, L149

Danilovic, S., Röhrbein, D., Cameron, R., \& Schüssler, M. 2012, A\&A, submitted

Danilovic, S., Schüssler, M., \& Solanki, S. K. 2010b, A\&\&A, 513, A1

Deinzer, W., Hensler, G., Schüssler, M., \& Weisshaar, E. 1984, A\&AA, 139, 426

Fan, Y. 2009, Living Rev. Solar Phys., 6, 4, http://www.livingreviews.org/lrsp-2009-4

Fang, F., Manchester, W., Abbett, W. P., \& van der Holst, B. 2010, ApJ, 714, 1649

Fang, F., Manchester, IV, W., Abbett, W. P., \& van der Holst, B. 2012, ApJ, 745, 37

Freytag, B., Steffen, M., Ludwig, H.-G., Wedemeyer-Böhm, S., Schaffenberger, W., \& Steiner, O. 2012, J. Comp. Phys., 231, 919

Gilman, P. A. \& Miller, J. 1981, ApJ Suppl., 46, 211

Gizon, L. \& Birch, A. C. 2012, PNAS, 109, 11896

Glatzmaier, G. A. 1985, ApJ, 291, 300 
Gudiksen, B. V., Carlsson, M., Hansteen, V. H., Hayek, W., Leenaarts, J., \& Martínez-Sykora, J. 2011, A\&BA, 531, A154

Hanasoge, S. M., Duvall, Jr., T. L., \& DeRosa, M. L. 2010, ApJ (Letters), 712, L98

Hanasoge, S. M., Duvall, Jr., T. L., \& Sreenivasan, K. R. 2012, PNAS, 109, 11928

Heinemann, T., Nordlund, Å., Scharmer, G. B., \& Spruit, H. C. 2007, ApJ, 669, 1390

Jacoutot, L., Kosovichev, A. G., Wray, A., \& Mansour, N. N. 2008, ApJ (Letters), 684, L51

Joshi, J., Pietarila, A., Hirzberger, J., Solanki, S. K., Aznar Cuadrado, R., \& Merenda, L. 2011, ApJ (Letters), 734, L18

Keller, C. U., Schüssler, M., Vögler, A., \& Zakharov, V. 2004, ApJ, 607, L59

Khomenko, E. \& Collados, M. 2012, ApJ, 747, 87

Kitiashvili, I. N., Kosovichev, A. G., Mansour, N. N., Lele, S. K., \& Wray, A. A. 2012a, Phys. Scr., 86, 018403

Kitiashvili, I. N., Kosovichev, A. G., Mansour, N. N., \& Wray, A. A. 2012b, ApJ (Letters), 751, L21

Kitiashvili, I. N., Kosovichev, A. G., Wray, A. A., \& Mansour, N. N. 2010, ApJ, 719, 307

Lagg, A., Solanki, S. K., Riethmüller, T. L., Martínez Pillet, V., Schüssler, M., Hirzberger, J., Feller, A., Borrero, J. M., Schmidt, W., del Toro Iniesta, J. C., Bonet, J. A., Barthol, P., Berkefeld, T., Domingo, V., Gandorfer, A., Knölker, M., \& Title, A. M. 2010, ApJ (Letters), 723, L164

Ludwig, H.-G., Caffau, E., Steffen, M., Freytag, B., Bonifacio, P., \& Kučinskas, A. 2009, Mem. Soc. Astr. It., 80, 711

Martínez-Sykora, J., De Pontieu, B., \& Hansteen, V. 2012, ApJ, 753, 161

Martínez-Sykora, J., Hansteen, V., \& Carlsson, M. 2008, ApJ, 679, 871

—. 2009, ApJ, 702, 129

Meneguzzi, M., Frisch, U., \& Pouquet, A. 1981, Phys. Rev. Let., 47, 1060

Moll, R., Cameron, R. H., \& Schüssler, M. 2011a, A\&A, 533, A126

-. 2012, A\&A, 541, A68

Moll, R., Pietarila Graham, J., Pratt, J., Cameron, R. H., Müller, W.-C., \& Schüssler, M. 2011b, ApJ, 736, 36

Muthsam, H. J., Kupka, F., Löw-Baselli, B., Obertscheider, C., Langer, M., \& Lenz, P. 2010, New Astron., 15, 460

Nordlund, A. 1983, in Solar Photosphere: Structure, Convection and Magnetic Fields, IAU Symp. 138, ed. J. O. Stenflo (Dordrecht: Reidel), 79

Nordlund, A. 1985a, Sol. Phys., 100, 209

Nordlund, A. 1985b, in Theoretical Problems High Resolution Solar Physics, ed. H. U. Schmidt, 101

Nordlund, A. \& Dravins, D. 1990, A\&A A, 228, 155

Nordlund, Å., Stein, R. F., \& Asplund, M. 2009, Living Rev. Solar Phys., 6, 2, http://www.livingreviews.org/lrsp-2009-2

Parker, E. N. 1963, ApJ, 138, 552

-. 1978, ApJ, 221, 368

Pietarila Graham, J., Cameron, R., \& Schüssler, M. 2010, ApJ, 714, 1606

Pietarila Graham, J., Danilovic, S., \& Schüssler, M. 2009, ApJ, 693, 1728

Rempel, M. 2011a, ApJ, 729, 5

-. 2011b, ApJ, 740, 15

-. 2012, ApJ, 750, 62

Rempel, M. \& Schlichenmaier, R. 2011, Living Rev. Solar Phys., 8, 3, http://www.livingreviews.org/lrsp-2011-3

Rempel, M., Schüssler, M., Cameron, R. H., \& Knölker, M. 2009a, Science, 325, 171

Rempel, M., Schüssler, M., \& Knölker, M. 2009b, ApJ, 691, 640

Robinson, F. J., Demarque, P., Li, L. H., Sofia, S., Kim, Y.-C., Chan, K. L., \& Guenther, D. B. 2003, MNRAS, 340, 923

Röhrbein, D., Cameron, R., \& Schüssler, M. 2011, A\& A, 532, A140

Sánchez Almeida, J. \& Martínez González, M. 2011, in ASP Conf. Ser. 437, Solar Polarization 
6, ed. J. R. Kuhn, D. M. Harrington, H. Lin, S. V. Berdyugina, J. Trujillo-Bueno, S. L. Keil, \& T. Rimmele, 451

Schaffenberger, W., Wedemeyer-Böhm, S., Steiner, O., \& Freytag, B. 2006, in ASP Conf. Ser. 354, Solar MHD Theory and Observations: A High Spatial Resolution Perspective, ed. J. Leibacher, R. F. Stein, \& H. Uitenbroek, 345

Scharmer, G. B., Henriques, V. M. J., Kiselman, D., \& de la Cruz Rodríguez, J. 2011, Science, 333,316

Schüssler, M., Shelyag, S., Berdyugina, S., Vögler, A., \& Solanki, S. K. 2003, ApJ, 597, L173

Schüssler, M. \& Vögler, A. 2006, ApJ (Letters), 641, L73

-. 2008, A\& A, 481, L5

Shelyag, S., Keys, P., Mathioudakis, M., \& Keenan, F. P. 2011, A\&A, 526, A5

Shelyag, S., Schüssler, M., Solanki, S. K., Berdyugina, S. V., \& Vögler, A. 2004, A $\& A, 427,335$

Shelyag, S., Schüssler, M., Solanki, S. K., \& Vögler, A. 2007, A\&GA, 469, 731

Spruit, H. C. 1976, Sol. Phys., 50, 269

-. 1979, Sol. Phys., 61, 363

Spruit, H. C. \& Zweibel, E. G. 1979, Sol. Phys., 62, 15

Stein, R. F. 2012, Living Rev. Solar Phys., 9, 4, http://www.livingreviews.org/lrsp-2012-4

Stein, R. F., Bercik, D., \& Nordlund, A. 2002, Nuovo Cimento C Geophys. Space Phys. C, 25, 513

Stein, R. F., Lagerfjärd, A., Nordlund, Å., \& Georgobiani, D. 2011, Sol. Phys., 268, 271

Stein, R. F. \& Nordlund, A. 1998, ApJ, 499, 914

Stein, R. F. \& Nordlund, A. 2006, ApJ, 642, 1246

—. 2012, ApJ (Letters), 753, L13

Steiner, O., Franz, M., Bello González, N., Nutto, C., Rezaei, R., Martínez Pillet, V., Bonet Navarro, J. A., del Toro Iniesta, J. C., Domingo, V., Solanki, S. K., Knölker, M., Schmidt, W., Barthol, P., \& Gandorfer, A. 2010, ApJ (Letters), 723, L180

Steiner, O., Grossmann-Doerth, U., Knölker, M., \& Schüssler, M. 1998, ApJ, 495, 468

Steiner, O., Grossmann-Doerth, U., Schussler, M., \& Knolker, M. 1996, Sol. Phys., 164, 223

Steiner, O. \& Rezaei, R. 2012, ArXiv e-prints, arXiv:1202.4040v1

Trujillo Bueno, J., Shchukina, N., \& Asensio Ramos, A. 2004, Nature, 430, 326

Ustyugov, S. D. 2010, Physica Scripta Vol. T, 142, 014031

Vögler, A. 2004, A\&A, 421, 755

Vögler, A., Shelyag, S., Schüssler, M., Cattaneo, F., Emonet, T., \& Linde, T. 2005, A $8 A, 429$, 335

Vargas Domínguez, S., Palacios, J., Balmaceda, L., Cabello, I., \& Domingo, V. 2011, MNRAS, 416,148

Vögler, A. 2003, PhD thesis, University of Göttingen, Germany, http://webdoc.sub.gwdg.de/diss/2004/voegler (in English)

Vögler, A. \& Schüssler, M. 2003, Astron. Nachr./AN, 324, 399

Vögler, A. \& Schüssler, M. 2007, A\& A, 465, L43

Webb, A. R. \& Roberts, B. 1978, Sol. Phys., 59, 249

Wedemeyer, S., Freytag, B., Steffen, M., Ludwig, H.-G., \& Holweger, H. 2004, A\&SA, 414, 1121

Wedemeyer, S., Ludwig, H.-G., \& Steiner, O. 2012, ArXiv e-prints, arXiv:1207.2342

Wedemeyer-Böhm, S. 2010, Mem. Soc. Astr. It., 81, 693

Wedemeyer-Böhm, S., Scullion, E., Steiner, O., Rouppe van der Voort, L., de La Cruz Rodriguez, J., Fedun, V., \& Erdélyi, R. 2012, Nature, 486, 505

Weiss, N. O. 1964, MNRAS, 128, 225

—. 1966, Proc. Roy. Soc. London, Ser. A, 293, 310

-. 2012, Geoph. Astrophys. Fluid Dyn., 106, 353

Yelles Chaouche, L., Cheung, M. C. M., Solanki, S. K., Schüssler, M., \& Lagg, A. 2009, A\&\&A, 507, L53

Yelles Chaouche, L., Moreno-Insertis, F., Martínez Pillet, V., Wiegelmann, T., Bonet, J. A., Knölker, M., Bellot Rubio, L. R., del Toro Iniesta, J. C., Barthol, P., Gandorfer, A., Schmidt, W., \& Solanki, S. K. 2011, ApJ (Letters), 727, L30 